Article

\title{
Molecular Dynamics Simulation on the Deposition Characteristics between Pt Cluster and Ni Substrate in Cold Gas Dynamic Spraying
}

\author{
Xueqing Zhang ${ }^{1, * \mathbb{C}}$, Xiaojing $\mathrm{Li}^{2}$ and Tianzhe Shi ${ }^{2}$ \\ 1 School of Energy and Power Engineering, North University of China, Taiyuan 030051, China \\ 2 Shanxi Qingze Sunshine Environmental Protection Technology Co., Ltd., Taiyuan 030051, China; \\ 1xj_cn@outlook.com (X.L.); sxqzyghb@163.cm (T.S.) \\ * Correspondence: xueqingzhang@nuc.edu.cn
}

check for updates

Citation: Zhang, X.; Li, X.; Shi, T. Molecular Dynamics Simulation on the Deposition Characteristics between Pt Cluster and Ni Substrate in Cold Gas Dynamic Spraying. Coatings 2022, 12, 142. https:// doi.org/10.3390/coatings12020142

Academic Editor: George Kokkoris

Received: 29 November 2021

Accepted: 23 January 2022

Published: 25 January 2022

Publisher's Note: MDPI stays neutral with regard to jurisdictional claims in published maps and institutional affiliations.

Copyright: (C) 2022 by the authors. Licensee MDPI, Basel, Switzerland. This article is an open access article distributed under the terms and conditions of the Creative Commons Attribution (CC BY) license (https:// creativecommons.org/licenses/by/ $4.0 /)$.

\begin{abstract}
During the process of cold spraying, the motion behavior and the arrangement of clusters, before impacting the substrate, have great influences on the coating/substrate bonding strength and the coating morphologies. In this work, the scattering and self-rotating movement of a single cluster and the different spatial positions of two clusters were taken into account to analyze the deposition characteristics between Pt clusters and Ni substrate by using the molecular dynamics method. We found that an excessively high normal velocity results in the failure of mechanical interlocking. Meanwhile, the increasing tangential velocity mainly enhances the mechanical interlocking. Moreover, the mechanical interlocking and the metallurgic bonding always are enhanced by increasing the impact torque around $x$-axis, but the metallurgic bonding increases only if the impact torque around $z$-axis is beyond a certain value. The results further show that, for the two neighboring clusters arranged horizontally, the thermal-softening effect of the first cluster impacting onto the substrate contributes more to its own metallurgic bonding and the mechanical interlocking of the latter one. In addition, for the two vertical clusters colliding with each other during their flying course, the smaller velocity difference can largely enhance the metal interlocking and the metallurgic bonding by shortening the cooling and solidifying times.
\end{abstract}

Keywords: cold gas dynamic spraying; molecular dynamics simulation; bonding mechanism; impacting parameters

\section{Introduction}

The thermal barrier coating (TBC) and oxidation-resistant coating of the Ni-base superalloy are widely applied in the hot sections of gas turbine engines, and the element $\mathrm{Pt}$ has become essential in these coatings [1,2]. To fabricate the Pt coating, the cold gas dynamic spraying (CGDS), a new surface treatment technology, was used in our laboratory. In the cold spraying, the micro or nano Pt particles are accelerated to 300-1200 m/s [3,4] by the supersonic gas stream through a converging-diverging nozzle and then deposited onto a metal substrate. The physical and chemical properties of the deposited particles can remain during the process of cold spraying because of its solid-state low-oxidized nature [5].

The mechanism of bonding in cold spraying is still a matter of some debate. Two different types of bonding mechanisms are proposed, namely the mechanical interlocking and the metallurgical bonding. The bonding and deposition were dominantly controlled by particle plasticity in the soft/hard system, and the physical trapping and mechanical interlocking played an important role for the hard/soft system [6]. Through assessing the bonding strength between the $\mathrm{Cu}$ coating and $\mathrm{Al}$ substrate, the mechanical interlocking was able to explain a large proportion of the total bonding strength, with metallurgical bonding only contributing significantly when the substrate had been polished and annealed prior to 
spraying [7]. The physical bonding phenomenon, according to the adiabatic shear instability at the $\mathrm{Al} / \mathrm{Ti}$ interface, could be associated with the chemical bonding and formation of an intermetallic layer that were aided by the thermo-mechanical processing and the deformation-assisted solid-state diffusion-based reactions [8]. No metallurgical bonding took place in a single-particle (Ni) impacting the substrate $(\mathrm{Al})$, but the metallurgical bonding was observed at the full coating deposition [9]. The strain-induced melting, phase transformation of dynamic amorphization, dynamic recrystallization and oriented crystallization from the prior-formed amorphous were considered the possible bonding mechanism of cold-spray coatings [10]. The copper particles deposited onto an aluminum substrate resulted in significant substrate deformation, which displayed a higher degree of metallurgical bonding at the coating/substrate interface [11]. The increased interfacial temperature significantly enhanced the coating $(\mathrm{Ni})$-substrate $(\mathrm{Cu})$ atomic mixture and the thermal softening effect. The former could improve the metallurgical bonding, and the latter could result in the prominent metal jet and strong mechanical interlock at the interface [12]. For cold spraying nickel (Ni) particles onto aluminum (Al) substrates, the adhesion strength did not always increase as a function of the particle preheating temperature. The remaining oxides film debris at the interface obstructed the formation of metallurgical bonding between particles and substrates, thus significantly weakening the bonding strength [13].

Molecular dynamics (MD) simulations offer possibilities to understand the CGDS bonding mechanisms at an atomistic level under the conditions of some macro experimental parameters [14-29], which will verify these experimental parameters through pure theoretical methods, without any uncontrollable factor, and optimize or simplify the existing experiments. Higher incident velocities, larger particle sizes and higher temperatures could improve the overall bonding strength between the clusters and substrates, such as the elements $\mathrm{Cu}$ and $\mathrm{Au}[14,15]$, and $\mathrm{Ni}$ and $\mathrm{Ti}$ [16]. The surface alloys were formed through exchanging sites between the cluster and substrate atoms, and the cluster atoms were rearranged following the substrate lattice arrangement from the bottom-up in the deposition process as the deposition process of Pd clusters was studied [17]. The study found that the quality of deposition was highest for an impact velocity of 500-700 m/s, the particle size of $20 \AA$ and an impact angle of $90^{\circ}[18,19]$. The outward flow in the particle (Al), followed by extensive deformation and the upward flow of the substrate (Al) in contact with the particle were firstly observed by the quasi-coarse-grained dynamics (QCGD) method [20], which implied the formation of metallurgical bonding. Comparing a particle of $2 \mathrm{~nm}$ size with one of $20 \mathrm{~nm}$ size at higher velocities $(>500 \mathrm{~m} / \mathrm{s})$, we see that the deformation of particles in the elastic region was followed by strong plastic behavior between Ti nanoparticles and Ti substrate, and it was attributed to the very high temperatures [21]. The effects of particle shapes, such as conical, cubic, cylindrical and spherical, on bonding mechanisms were also studied by Hesamodin and Ahmad [22]. The local disorder and large atomic displacements provided an indication of fluid-like behavior from the center of contact region towards edges, as the $\mathrm{Cu}$ particles with the varied diameter from 5 to $40 \mathrm{~nm}$ were accelerated against the $\mathrm{Cu}$ substrate [23]. The higher bonding strength and compatibility of $\mathrm{Pd}$ and $\mathrm{Cu}$ were expressed by the larger interfacial bonding energy and interfacial shearing strength at the $\mathrm{Pd}-\mathrm{Cu}$ composite metal membrane (CMM) interface [24]. The bonding formation mechanism for nanoscale ceramic particle $\left(\mathrm{TiN}\right.$ or $\left.\mathrm{TiO}_{2}\right)$ collision was largely determined by the relationship between the adhesion energy and the rebound energy, and the nanoscale ceramic particles could be bonded together, as the adhesion energy was higher than the rebound energy [25-27]. Recently, the metallurgical bonding mechanism of the nano-scale ceramic particle $\left(\mathrm{TiO}_{2}\right)$ was verified by the atomic displacement and lattice distortion, which was specifically due to the debonding of oxygen atoms from $\mathrm{TiO}_{2}$ and bonding with $\mathrm{Ti}$ atoms in the substrate $[28,29]$. 
The effects of the impact angle, the particle rotation and the peening strength on the bonding mechanism have been investigated in the previous works. The contact area between the deformed particle and substrate decreased, and the crater depth in the substrate was reduced by increasing the tilting angle at the same impact velocity [30]. The bonding strength between the $\mathrm{Al}$ particle and $\mathrm{Al}$ substrate increased with decreasing spray angle from the normal direction ( $90^{\circ}$ spray angle), and the maximum bonding strength was observed at $45^{\circ}$ spray angle, but the deposition efficiency decreased with decreasing spray angle [31]. The dimple-like features, which are a sign of metallurgical bonding, were formed in the surrounding area of the central region for different spray angles in the perpendicular spray [32]. The jet of particles perpendicular to the substrate (at $90^{\circ}$ ) had thicker coatings compared to the $60^{\circ}$ angle of impact, but more uniform coatings were observed at the impact angle of $60^{\circ}[18,19]$. The three-dimensional model considering the rotation and translation of particles resulted in more precise deformation and bonding compared to the 2D model [33]. The strengthened peening effect by the successive impacts of the particle with higher kinetic energy was beneficial for the formation of metallurgical bonding [9]. Large stainless-steel particles were introduced into the spray powders as in situ shot peen particles to create a dense $\mathrm{Al}$ deposit and to improve the bond strength of joints [34].

From the literature reviews, it is evident that the effects of some impact parameters (including the impacting velocity and torque for a single cluster and the interactions of two neighboring clusters) on the bonding strength and the real complex phenomenon of clustersubstrate and cluster-cluster during the CGDS process have not been well investigated in detail at the atomic levels. Thus, in the present work, MD simulations were performed to comprehensively study the bonding mechanism during the process of CGDS and to understand in depth the effects of the impact parameters on the deposition characteristics during the CGDS process between the Pt clusters and Ni substrate. Three models were built: (i) Model 1, one single cluster with the different incident velocities and the different impact torques; (ii) Model 2, two clusters arranged horizontally with the different incident velocity and the various distances between them; and (iii) Model 3, two clusters arranged vertically with the different incident velocities.

\section{Materials and Methods}

The simulation system consisted of a platinum $(\mathrm{Pt})$ cluster and a nickel $(\mathrm{Ni})$ substrate. The cluster was composed of 140 atoms arranged in the face-centered cubic (fcc) lattice structure, with a lattice spacing of $3.92 \AA$, and its diameter was $15.68 \AA$. The substrate was made of $71,680 \mathrm{Ni}$ atoms arranged in the fcc lattice structure, with a lattice spacing of $3.52 \AA$, and its dimensions were $112.64 \AA \times 112.64 \AA \times 56.32 \AA$. The cross-sectional views of 3D models in the $x-z$ plane are shown in Figure 1. The top layer of the substrate was the free layer to reflect the bombardment process, the middle layer acted as the thermal control layer and maintained a constant temperature of $300 \mathrm{~K}$ by the Nose-Hoover method [35] and the bottom layer was the fixed layer to hinder the movement of the substrate mass center caused by the bombardment of incident clusters. The simulated parameters are given in Table 1.

MD simulations of the CGDS process were performed by using "Large-scale atomic/ molecular massively parallel simulator" (LAMMPS) [36]. The velocity Verlet algorithm with a time step of $0.001 \mathrm{ps}$ was employed to solve the Newton motion equation of the atoms. The interactions among the atoms were described by using Embedded Atom Method (EAM) [37], which was a suitable interatomic potential for obtaining the structural, mechanical and thermal properties of alloy systems, including $\mathrm{Ni}$ and Pt. In the EAM scheme, the total energy of a collection of atoms is calculated by using the following:

$$
\mathrm{E}=\frac{1}{2} \sum_{i, j, i \neq j} \phi_{i j}\left(r_{i j}\right)+\sum_{i} F_{i}\left(\rho_{i}\right)
$$


where $\phi_{i j}$ corresponds to the pairwise interaction between atoms $i$ and $j$ separated by the distance $r_{i j} ; F_{i}$ is the energy resulted from embedding atom $i$ into a local site with the electron density, $\rho_{i}$, which is calculated by using the following equation:

$$
\rho_{i}=\sum_{j, j \neq i} f_{j}\left(r_{i j}\right)
$$

here $f_{j}\left(r_{i j}\right)$ is the electron density at the site of atom $i$ arising from atom $j$ at a distance $r_{i j}$ away. In the simulation, the cutoff distance of $6.0 \AA$ was used to improve the computation efficiency.

At the starting point, the cluster was located at a sufficient position above the substrate surface to avoid the interaction between them. Each system was equilibrated for 1000 ps, using an NVT ensemble (at $300 \mathrm{~K}$ by Nose-Hoover method [35]), before impacting, and $1000 \mathrm{ps}$ run in the NVE ensemble to collect the data. In practical simulations, the computational time for each case needed $8 \mathrm{~h}$ or so at a four-core CPU. Periodic boundary conditions were applied in both $\mathrm{x}$ and $\mathrm{y}$ directions, and the boundary condition in $\mathrm{z}$ direction was non-periodic.

Table 1. Parameters used in the MD simulation of CGDS process.

\begin{tabular}{|c|c|c|}
\hline \multirow[b]{2}{*}{ Materials } & Substrate Material & Ni, Cuboid $(112.64 \AA$ × $\times 112.64 \AA ̊ 56.32 \AA), 71,680$ Atoms \\
\hline & Cluster Material & Pt, Spherical Cluster (Diameter $d=15.68 \AA$ ), 140 Atoms \\
\hline \multirow{13}{*}{$\begin{array}{l}\text { Operating } \\
\text { Parameters }\end{array}$} & Bulk Temperature & $300 \mathrm{~K}$ \\
\hline & Potential Function & Embedded Atom Potential (EAM) [37] \\
\hline & Thickness of Fixed Layer & $k=3.52 \AA$ \\
\hline & Thickness of Thermostat Layer & $l=24.64 \AA$ \\
\hline & Thickness of Free Layer & $m=28.16 \AA$ \\
\hline & Initial Stand-Off Distance & $n=62.72 \AA$ \\
\hline & Time Step & $0.001 \mathrm{ps}$ \\
\hline & Duration of Relaxation & 1000 ps \\
\hline & Duration of Simulation & $1000 \mathrm{ps}$ \\
\hline & $\begin{array}{l}\text { Incident Velocity of Cluster } 0 \text { in } \\
\text { Model } 1\end{array}$ & $\begin{array}{c}V_{x, 0}=0 \sim \\
200 \mathrm{~m} / \mathrm{s} \text { or } V_{z, 0}=300 \sim \\
900 \mathrm{~m} / \mathrm{s} .\end{array}$ \\
\hline & $\begin{array}{l}\text { Incident Torque of Cluster } 0 \text { in } \\
\text { Model } 1\end{array}$ & $V_{x, 0}=0 \mathrm{~m} / \mathrm{s}$ and $V_{z, 0}=600 \mathrm{~m} / \mathrm{s.} . \begin{array}{c}T_{x, 0}=1 \sim \\
100 \mathrm{ev} \text { or } T_{z, 0}=1 \sim 100 \mathrm{ev} .\end{array}$ \\
\hline & $\begin{array}{l}\text { Incident Velocity of Cluster } 1 \\
\text { and Cluster } 2 \text { in Model } 2\end{array}$ & \multirow{2}{*}{$\begin{array}{c}V_{z, 1}=300 \sim \\
900 \mathrm{~m} / \mathrm{s} \text { or } V_{z, 2}=300 \sim \\
900 \mathrm{~m} / \mathrm{s} . \\
T_{x, 0}=0 \text { ev and } T_{z, 0}=0 \mathrm{ev.} \quad 300 \sim \\
\quad V_{z, 3}=300 \mathrm{~m} / \mathrm{s} \text { or } V_{z, 4}=300 \sim \\
900 \mathrm{~m} / \mathrm{s} .\end{array}$} \\
\hline & $\begin{array}{l}\text { Incident Velocity of Cluster } 3 \\
\text { and Cluster } 4 \text { in Model } 3\end{array}$ & \\
\hline
\end{tabular}

Note: $V$ is the incident velocity; $T$ is the impact torque. The subscripts $\mathrm{x}, \mathrm{y}$, and $\mathrm{z}$ are the coordinate directions. The subscripts $0,1,2,3$, and 4 are the cluster numbers. For example, $T_{x, 0}$ is the torque component around the $x$-axis through Cluster 0 center of mass. 


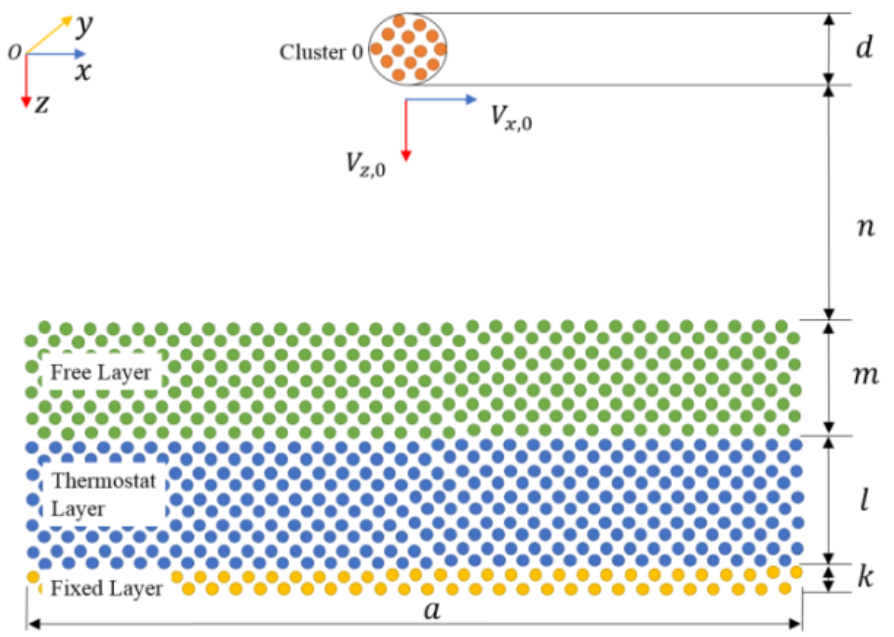

(a)

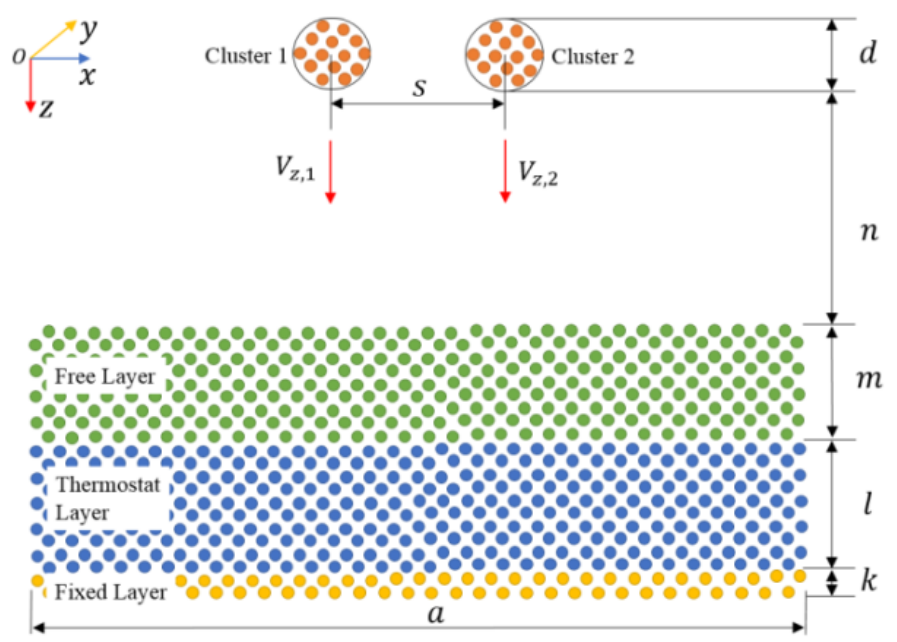

(b)

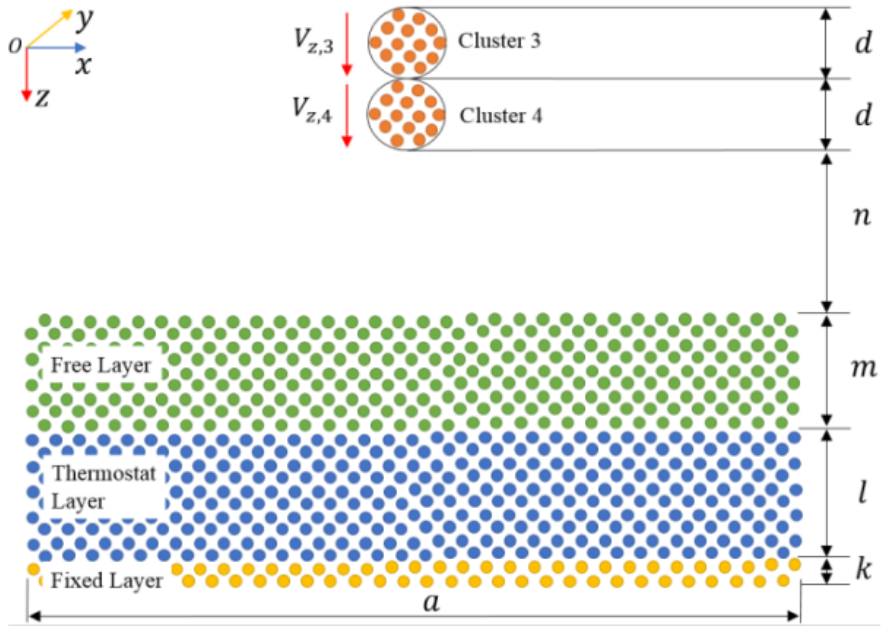

(c)

Figure 1. Cross-sectional views of 3D models in the $x-z$ plane: (a) Model 1, one single cluster; (b) Model 2, two horizontal clusters; and (c) Model 3, two vertical clusters. 


\section{Results and Discussion}

In order to characterize the deposition of Pt clusters onto Ni substrate during the process of CGDS, the normal velocity, the tangential velocity and the self-rotation (around the $x$-axis through the center of mass of the cluster in the $y-z$ plane and around the $z$-axis through the center of mass of cluster in the $x-y$ plane) were firstly simulated. Furthermore, the interactions between two clusters arranged horizontally and vertically in the depositing process were studied, respectively. Binding energy was employed to judge the bonding strength between the clusters and substrate, and a larger absolute value of binding energy suggested a higher bonding strength [24-26,38-40]. The binding energy of this paper was compared with that in the literature $[25,26]$ to verify the reliability of MD simulations and that their trends were in good agreement, as shown in Figure 2. Additionally, the meansquared displacement (MSD) of atoms inside the free layer was an important parameter to reflect the plastic deformation of the substrate, and a larger value of MSD implied the formation of metallurgical bonding, due to the particles' deeper embedding into the substrate [23]. The local atomic density could be described by the radial distribution function (RDF, also called $g(r)$ ), and the value of peak is used to characterize the order degree of the crystal-structural properties, as this was usually viewed as a sign of the metallurgic bonding $[14,15,17,24]$.

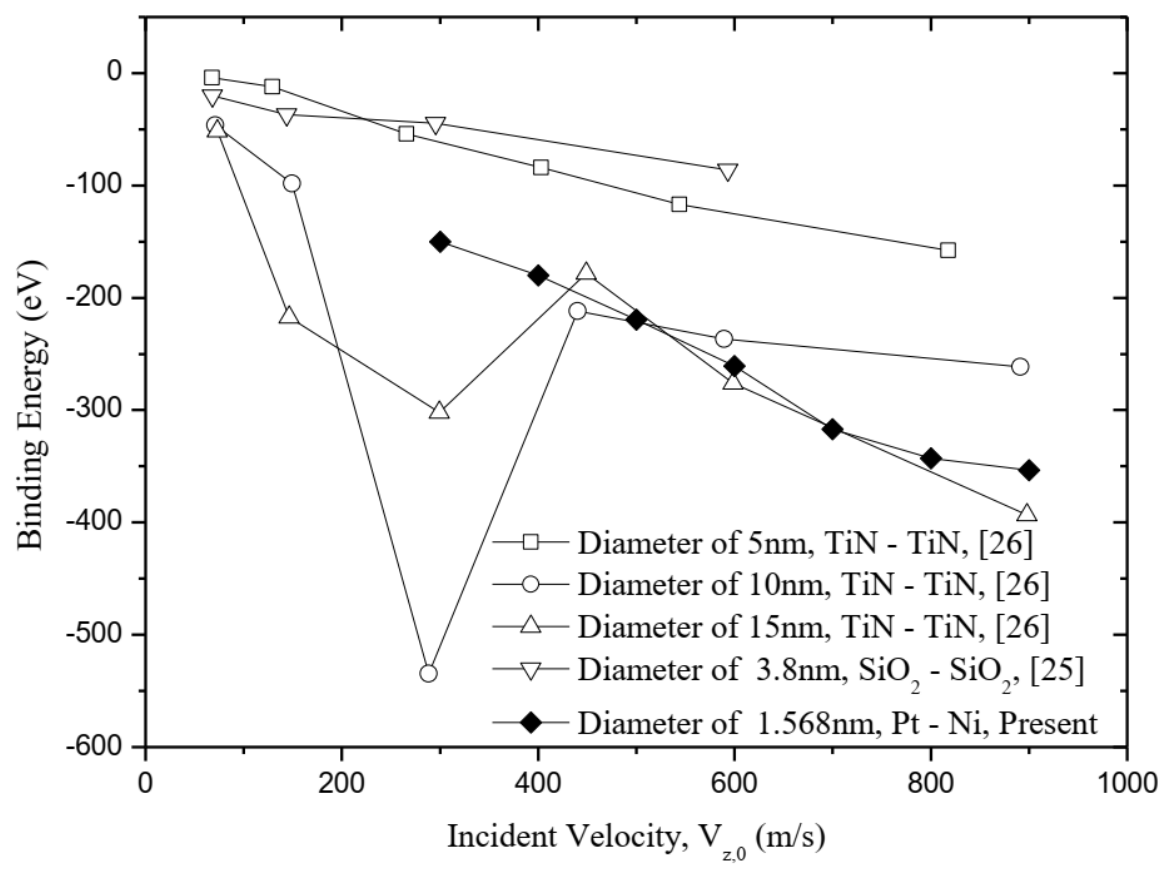

Figure 2. Comparison of binding energy between the present results and those of the literature $[25,26]$.

\subsection{A single Cluster at the Different Incident Velocity in Model 1}

Figure 3 shows the deposition morphologies at the different normal incident speeds, $V_{z, 0}$. The cluster does not embed into the substrate, and the coating is mainly made of the cluster material at the incident velocity of $300 \mathrm{~m} / \mathrm{s}$, as shown in Figure 3a. As the speed increases, the cluster embeds into the substrate with the different depths, and the coating is made of the composite of the cluster and the substrate material (see Figure 3b) or the full substrate material (see Figure 3c). It suggests that maintaining the incident speed within an optimal range is critical in order to achieve a uniform coating. Additionally, a larger $V_{z, 0}$ leads to the deeper embeddedness into substrate and the decrease in the deposition height. At the same time, as $V_{x, 0}$ increases, the embedding depth does not almost change, but more cluster atoms are scattered into the substrate, leading to the higher bonding strength (see Figure 4), because the bonding strength increases with the decreasing spray angle from the normal direction $\left(90^{\circ}\right.$ spray angle) $[18,19,31]$, which can been seen further from Figure 5 . 
It is noted that the spraying of clusters is not absolutely concentrated from the nozzle to the substrate in the real-world CGDS process, and the reciprocating motion of the nozzle or the substrate along $\mathrm{x}$ or $\mathrm{y}$ direction also brings about the spraying non-perpendicular to the substrate. Thus, the clusters more or less have a tangential velocity, resulting in the larger edge of spraying area, and the clusters in the center of the spraying area can achieve a higher deposition height, while the ones on the edge can bond stronger into the substrate.

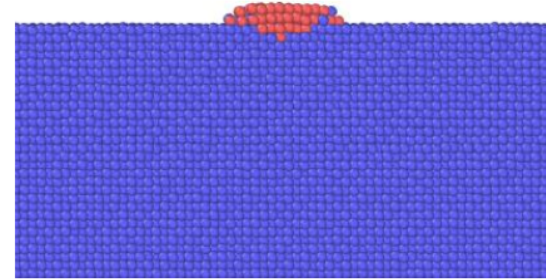

(a)

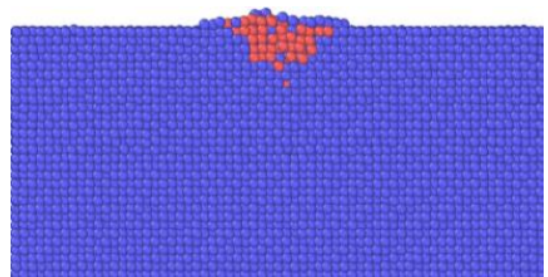

(b)

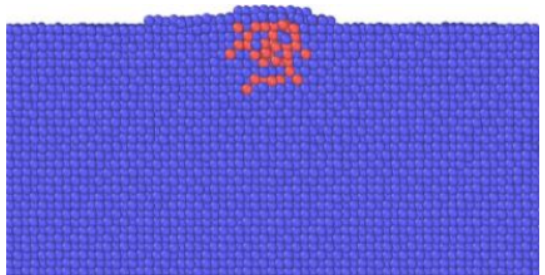

(c)

Figure 3. Morphology snapshots (side views of the center section along $x-z$ plane) of Cluster 0 and substrate after impacting at three different $V_{z, 0}$ in Model 1: (a) $V_{z, 0}=300 \mathrm{~m} / \mathrm{s}$, (b) $V_{z, 0}=600 \mathrm{~m} / \mathrm{s}$ and (c) $V_{z, 0}=900 \mathrm{~m} / \mathrm{s}$. In all cases, $V_{x, 0}=0 \mathrm{~m} / \mathrm{s}$.

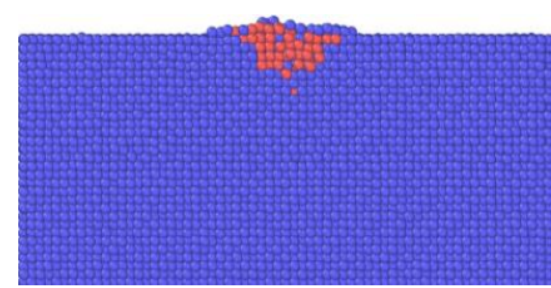

(a)

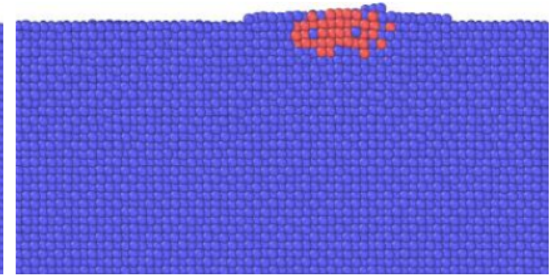

(b)

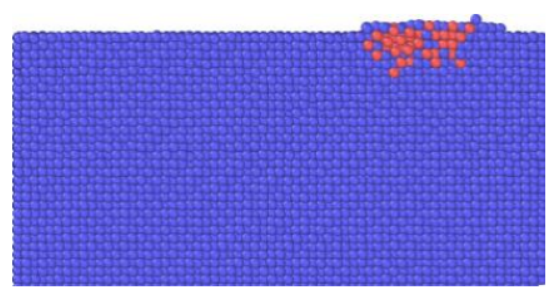

(c)

Figure 4. Morphology snapshots (side views of the center section along $\mathrm{x}-\mathrm{z}$ plane) of Cluster 0 and substrate after impacting at three different $V_{x, 0}$ in Model 1: (a) $V_{x, 0}=0 \mathrm{~m} / \mathrm{s}$, (b) $V_{x, 0}=100 \mathrm{~m} / \mathrm{s}$ and (c) $V_{x, 0}=200 \mathrm{~m} / \mathrm{s}$. In all cases, $V_{z, 0}=600 \mathrm{~m} / \mathrm{s}$.

Figure 5a displays the binding energy between the cluster and the free layer, and the MSD of the free layer obtained at the different $V_{z, 0}$ and $V_{x, 0}=0 \mathrm{~m} / \mathrm{s}$. It is observed that both the absolute values of binding energy and the MSD value increase at the larger $V_{z, 0}$. Moreover, the absolute value of binding energy increases the most as $V_{z, 0}$ varies in the range from 600 to $700 \mathrm{~m} / \mathrm{s}$, whereas the increment slightly decreases as $V_{z, 0}$ is higher than $700 \mathrm{~m} / \mathrm{s}$. In the meantime, the MSD increases the most in the range of $V_{z, 0}=700-900 \mathrm{~m} / \mathrm{s}$. Thus, the substrate deformation and the bonding strength are not always in sync when the impact velocity is over the critical range. Thus is because the shear instability takes place at $V_{z, 0}=600-700 \mathrm{~m} / \mathrm{s}$, and the mechanical interlocking gradually tends to fail at $V_{z, 0} \geq 700 \mathrm{~m} / \mathrm{s}$, due to the penetration. The same phenomenon is found in the literature [24-26]. In addition, it can be seen from Figure 5b that both the absolute values of binding energy and the MSD value increase with the rising $V_{x, 0}$, and their increments are fairly consistent. Thus, one higher tangential speed of the cluster can lead to a stronger bonding force between the cluster and the substrate, and this is attributed to the wider physical trapping in the substrate. 

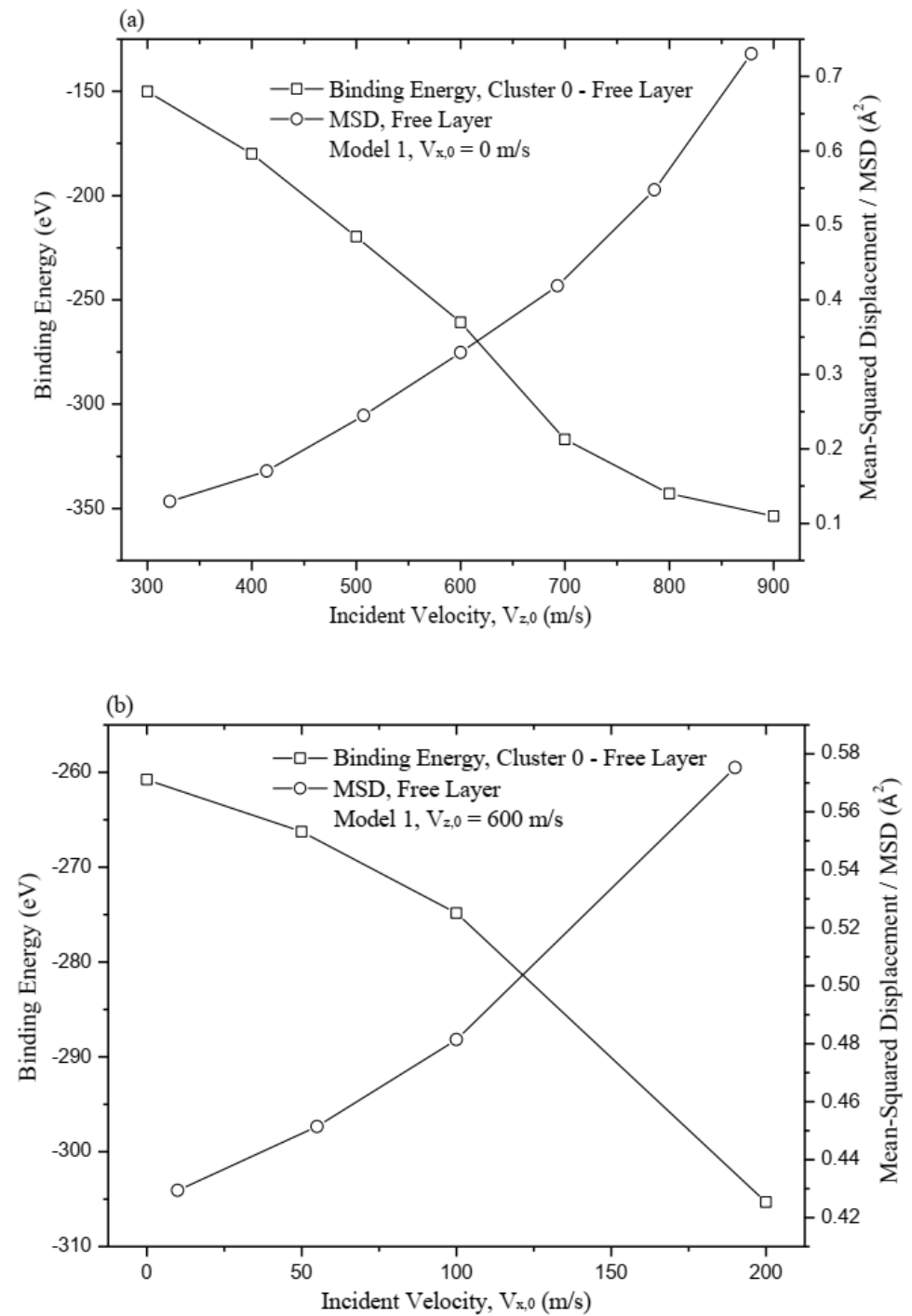

Figure 5. Binding energy between Cluster 0 and free layer and MSD of free layer in Model 1: (a) with different $V_{z, 0}$ at $V_{x, 0}=0 \mathrm{~m} / \mathrm{s}$ and (b) with different $V_{x, 0}$ at $V_{z, 0}=600 \mathrm{~m} / \mathrm{s}$.

The RDFs at the different $V_{x, 0}$ and $V_{z, 0}$ are plotted in Figure 6. The value of each peak becomes larger when the incident $V_{z, 0}$ increases (see Figure 6a), suggesting the crystal order degree of the whole system enhances after depositing with increasing $V_{z, 0}$. Thus, more metallic bonds are formed between $\mathrm{Pt}$ and Ni atoms and the metallurgic bonding is intensified, as is in line with the results in the literature $[14,15,17,24]$. However, the peak values almost keep the same as the tangential velocity increases in Figure $6 \mathrm{~b}$, meaning that the tangential velocity $\left(V_{x, 0}\right)$ has little or no effect on the formation of metallurgic bonding. 

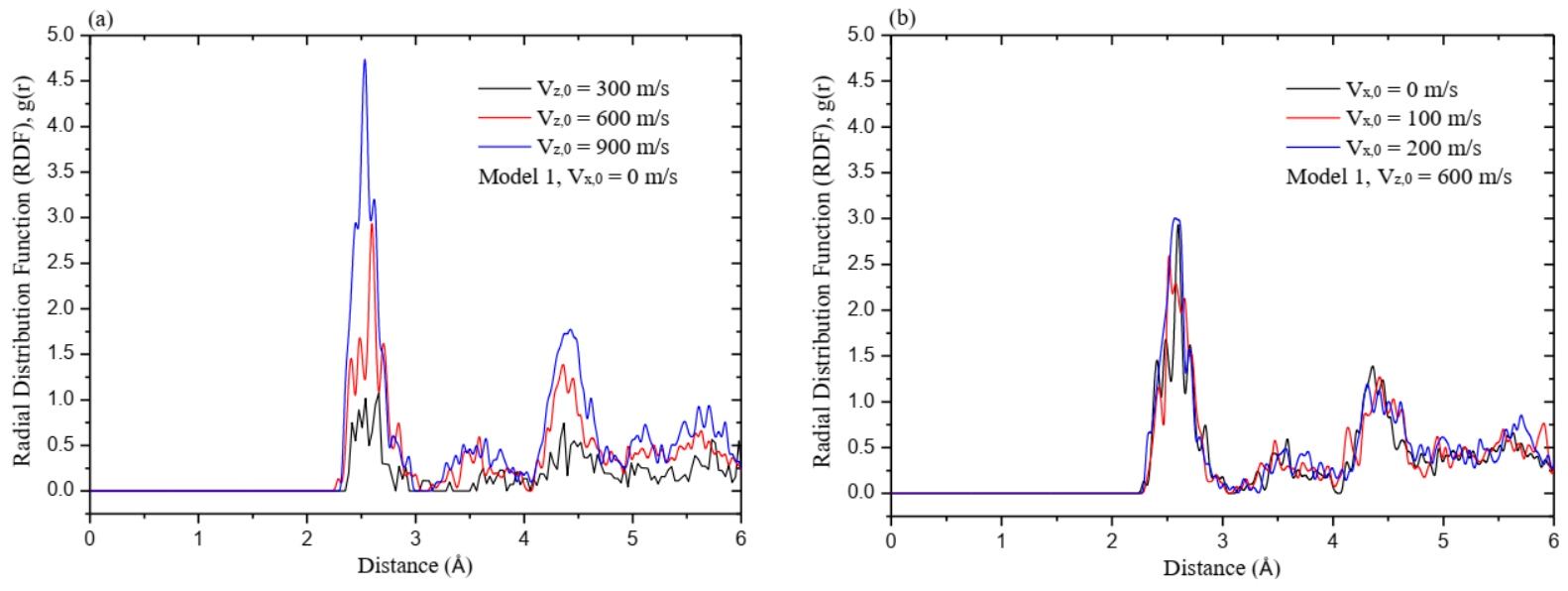

Figure 6. RDFs in Model 1: (a) $V_{x, 0}=0 \mathrm{~m} / \mathrm{s}$ and various $V_{z, 0}$ and (b) $V_{z, 0}=600 \mathrm{~m} / \mathrm{s}$ and various $V_{x, 0}$.

\subsection{A Single Cluster at the Different Impact Torques in Model 1}

When the clusters are fed into the spraying nozzle, they may rotate around their axes of mass in the accelerating and spraying process. Figure 7 represents the deposition morphologies at the different impact torque, $T_{x, 0}$, and $V_{z, 0}=600 \mathrm{~m} / \mathrm{s}$. From the figures, it can be observed that the embedding depth of the cluster into the substrate apparently increases with the larger $T_{x, 0}$, due to the centrifugal force. $T_{x, 0}$ can lead one part of $\mathrm{Pt}$ atoms to embed into the Ni substrate and the other ones to scatter into the substrate during the process of impacting. Since the incident velocity and the centrifugal force are in the same plane for a single atom, the phenomenon of Pt atoms scattering into the Ni substrate exists, but the scattering area is not very large. Consequently, a more remarkable thermalsoftening effect takes place on the substrate than that without the impact torque. Thus, the depth of the whole cluster embedding into the substrate largely increases. Additionally, the scattering area significantly enlarges at the larger $T_{z, 0}$, but the embedding depth hardly changes, as shown in Figure 8. Because the centrifugal force is always perpendicular to the initial incident velocity, $\mathrm{Pt}$ atoms finally spray into Ni substrate in the diverging pattern. Moreover, the weakened thermal-softening effect caused by scattering cannot contribute more to the embedding depth.

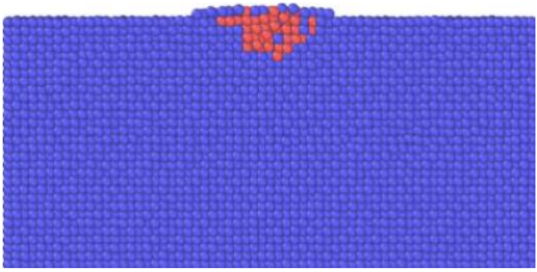

(a)

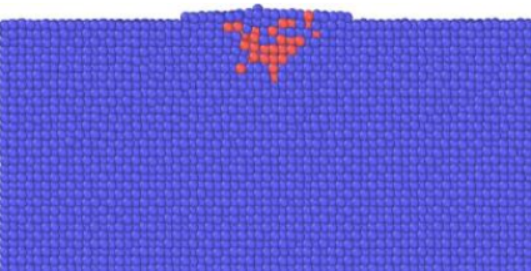

(b)

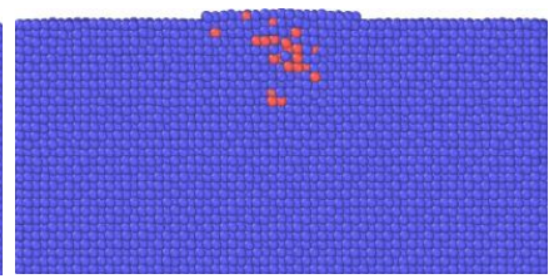

(c)

Figure 7. Morphology snapshots (side views of the center section along $\mathrm{x}-\mathrm{z}$ plane) of Cluster 0 and substrate after impacting at the different $T_{x, 0}$ in Model 1: (a) $T_{x, 0}=1 \mathrm{ev},(\mathbf{b}) T_{x, 0}=50 \mathrm{ev}$ and (c) $T_{x, 0}=100 \mathrm{ev}$. In all cases, $V_{z, 0}=600 \mathrm{~m} / \mathrm{s}$ and $T_{z, 0}=0 \mathrm{ev}$. 


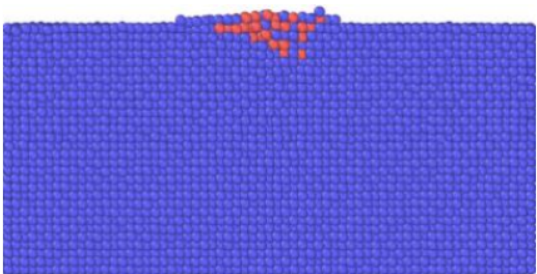

(a)

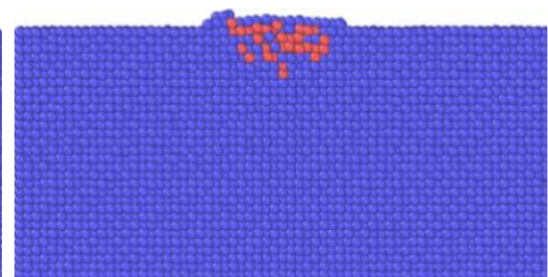

(b)

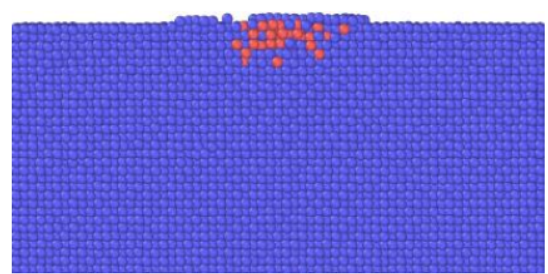

(c)

Figure 8. Morphology snapshots (side views of the center section along $\mathrm{x}-\mathrm{z}$ plane) of Cluster 0 and substrate after impacting at the different $T_{z, 0}$ in Model 1: (a) $T_{z, 0}=1 \mathrm{ev},(\mathbf{b}) T_{z, 0}=50 \mathrm{ev}$ and (c) $T_{z, 0}=100 \mathrm{ev}$. In all cases, $V_{z, 0}=600 \mathrm{~m} / \mathrm{s}$ and $T_{x, 0}=0 \mathrm{ev}$.

The changes in the binding energy between Cluster 0 and the free layer with the impact torque are plotted in Figure 9a. The absolute value of the binding energy between the cluster and the free layer presents a remarkably increasing trend, as a larger $T_{x, 0}$ is considered. Thus, the self-rotation around $x$-axis can always enhance the bonding strength, which is attributed to the increase in the mechanical interlocking and metallurgic bonding induced by the thermal-softening effect on the substrate. Meanwhile, the absolute value of the binding energy firstly decreases and then increases with the increasing $T_{z, 0}$. Although the mechanical interlocking becomes stronger with Pt atoms scattering into Ni substrate in the $x-y$ plane, the metallurgic bonding is first weakened and then intensified. Comparing the symbols $\times$ and + , the impact torque around $z$-axis plays a dominant role in the bonding force, as $T_{z, 0}$ and $T_{x, 0}$ are both less than $10 \mathrm{eV}$, while the impact torque around $x$-axis improves the bonding strength more as $T_{z, 0}$ and $T_{x, 0}$ are both higher than $10 \mathrm{eV}$.
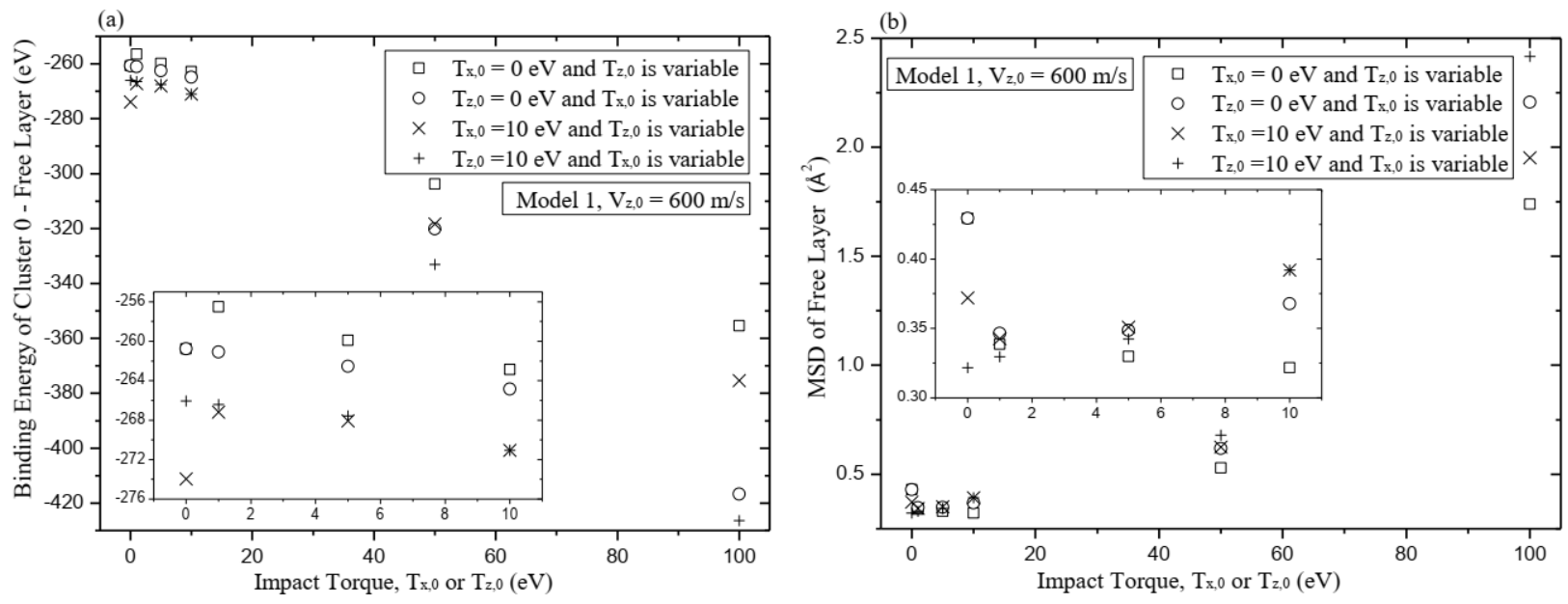

Figure 9. (a) Binding energy between Cluster 0 and free layer at the different $T_{x, 0}$ and $T_{z, 0}$. (b) MSD of free layer at different $T_{x, 0}$ and $T_{z, 0}$. All cases are in Model 1, and $V_{z, 0}=600 \mathrm{~m} / \mathrm{s}$.

Figure $9 \mathrm{~b}$ shows that the smaller $T_{x, 0}$ and $T_{z, 0}$ can both reduce MSD, as compared to the cases without the impact torque, but the larger $T_{x, 0}$ and $T_{z, 0}$ contribute to the larger ones, and the effect that resulted from the increasing $T_{x, 0}$ is more remarkable. The centrifugal rotation of the cluster makes some $\mathrm{Pt}$ atoms scatter and embed into the space between the different $\mathrm{Ni}$ atoms in a single atomic pattern, of course, which results in the smaller deformation of the substrate than a bulk of Pt atoms impacting the Ni substrate without the impact torque. However, the deformation of the substrate significantly enlarges with more $\mathrm{Pt}$ atoms scattering into the Ni substrate and the longer scattering path when $T_{x, 0}$ and $T_{z, 0}$ increase and are beyond a certain value. As it can be seen in Figure $9 \mathrm{~b}$, the impact torque around the $x$-axis has a more significant effect on the substrate deformation than the 
one around the z-axis. It is attributed to the outstanding thermal-softening effect when the centrifugal movement and the incident velocity of $\mathrm{Pt}$ atoms are in the same plane.

Figure 10 shows the RDFs after the clusters impacting the substrate at the different $T_{x, 0}$ and $T_{z, 0}$. The peak values of RDF are higher the with increasing $T_{x, 0}$ in Figure 10a, indicating that the centrifugal movement of $\mathrm{Pt}$ atoms in the $\mathrm{y}-\mathrm{z}$ plane always has a positive effect on the order degree of crystal. It can give rise to a stronger metallurgic bonding. However, as $T_{z, 0}$ is increasing, the peak values of RDF first decrease and then increase in Figure 10b. Thus suggests that the metallurgic bonding increases only if $T_{z, 0}$ is beyond a certain value. Comparing Figure 10a with Figure 10b, we can conclude that the mechanical interlocking dominates the bonding strength at a smaller $T_{z, 0}$.
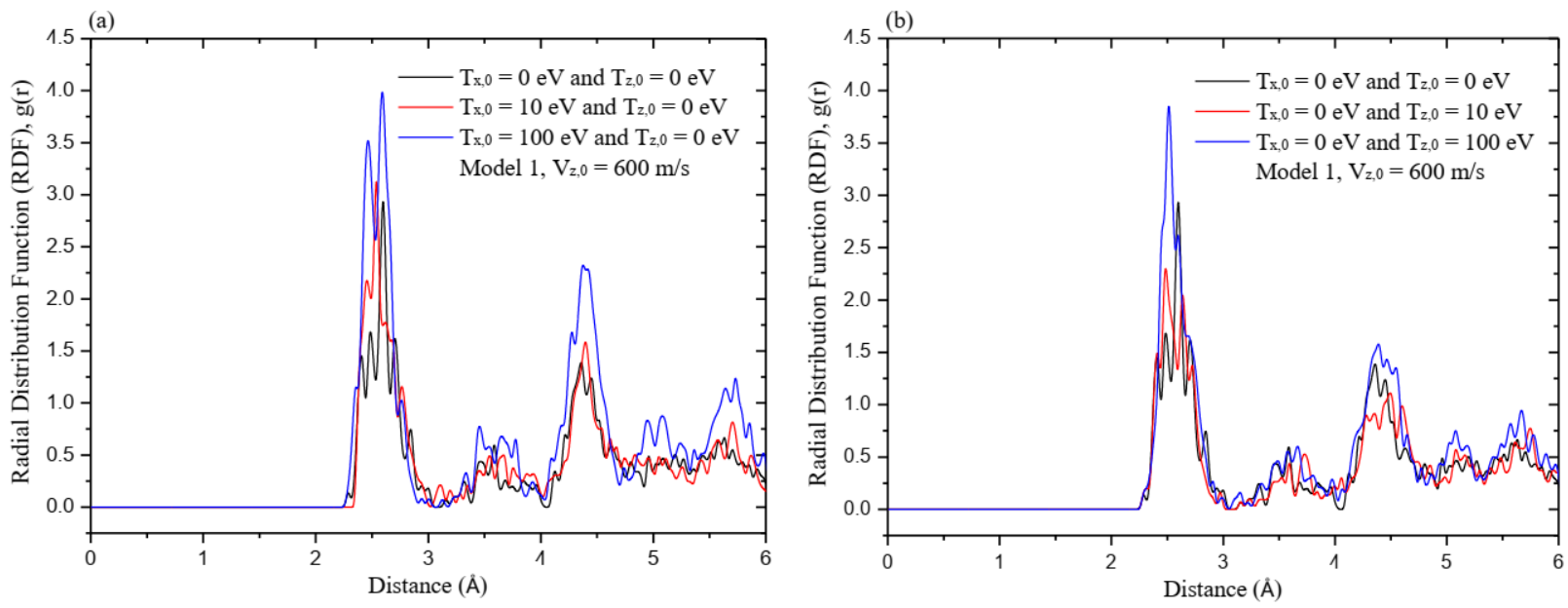

Figure 10. RDFs in Model 1: (a) $T_{z, 0}=0 \mathrm{eV}$ and various $T_{x, 0}$ and (b) $T_{x, 0}=0 \mathrm{eV}$ and various $T_{z, 0}$.

\subsection{Two Clusters Arranged Horizontally at the Different Incident Velocities in Model 2}

Figure 11a shows that the absolute value of the binding energy between each cluster and the free layer obviously increases with the increasing $V_{z, 2}$, but the binding energy between the two clusters gradually declines at $V_{z, 2}>700 \mathrm{~m} / \mathrm{s}$. This implies that the bonding force between each cluster and the substrate becomes stronger, as any one of the two clusters is at a higher incident velocity. It is due to the improved mechanical interlocking and metallurgic bonding induced by the thermal-softening effect from the cluster first impacting the substrate. Comparing the symbols $\square$ with $\bigcirc$, it can be observed that the bonding strength between Cluster 2 and the substrate is always larger than the one between Cluster 1 and the substrate with increasing $V_{z, 2}$, even when the incident velocity of Cluster 2 is smaller than that of Cluster $1\left(V_{z, 2}<600 \mathrm{~m} / \mathrm{s}\right)$. Thus, the bonding strength is determined dominantly by the mechanical interlocking at $V_{z, 2}<600 \mathrm{~m} / \mathrm{s}$ but the metallurgic bonding at $V_{z, 2}>700 \mathrm{~m} / \mathrm{s}$. Since the top substrate is penetrated by Cluster 2 at $V_{z, 2}>700 \mathrm{~m} / \mathrm{s}$, its own mechanical interlocking fails, as was verified in Figure 3c. Therefore, when the two neighboring clusters arranged horizontally are impacting the substrate one after another, the thermal-softening effect from the first cluster contributes more to its own metallurgic bonding and the mechanical interlocking of the second cluster. The metallurgic bonding is formed in only a very short time and requires the atomic distance of Pt cluster and Ni substrate to be quite close, which is difficult to achieve, as the latter cluster impacts the softened substrate. The deformation rate of the substrate significantly increases at $V_{z, 2}>700 \mathrm{~m} / \mathrm{s}$ in Figure $11 \mathrm{~b}$, indicating the further failure of mechanical interlocking that is caused by the penetration of the substrate. Figure 12 displays that the crystal order degree of the whole system becomes larger with the increasing $V_{z, 2}$, which implies that the incident velocity of any one cluster improves the metallurgic bonding of the whole system. 

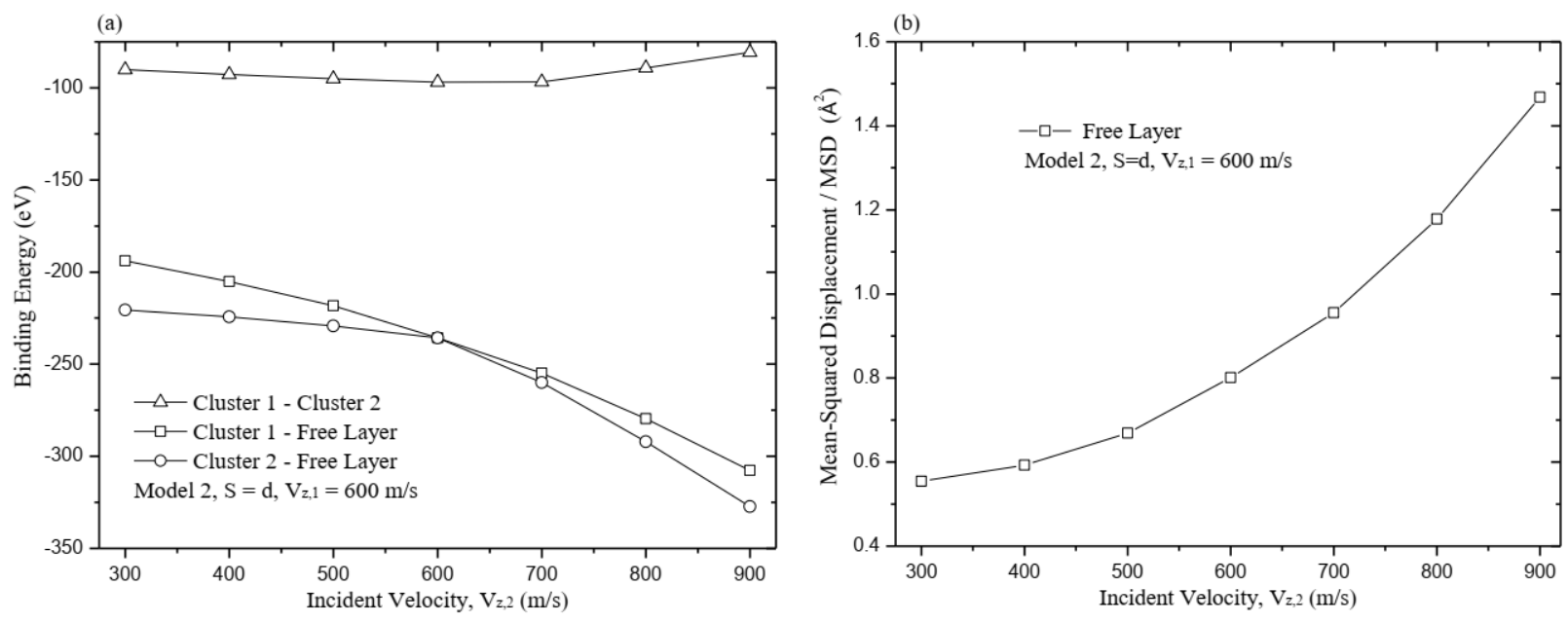

Figure 11. (a) Binding energy among Cluster 1, Cluster 2 and free layer at various $V_{z, 2} \cdot$ (b) MSD of free layer at various $V_{z, 2}$. In all cases, $V_{z, 1}=600 \mathrm{~m} / \mathrm{s}$ and $\mathrm{S}=\mathrm{d}$.

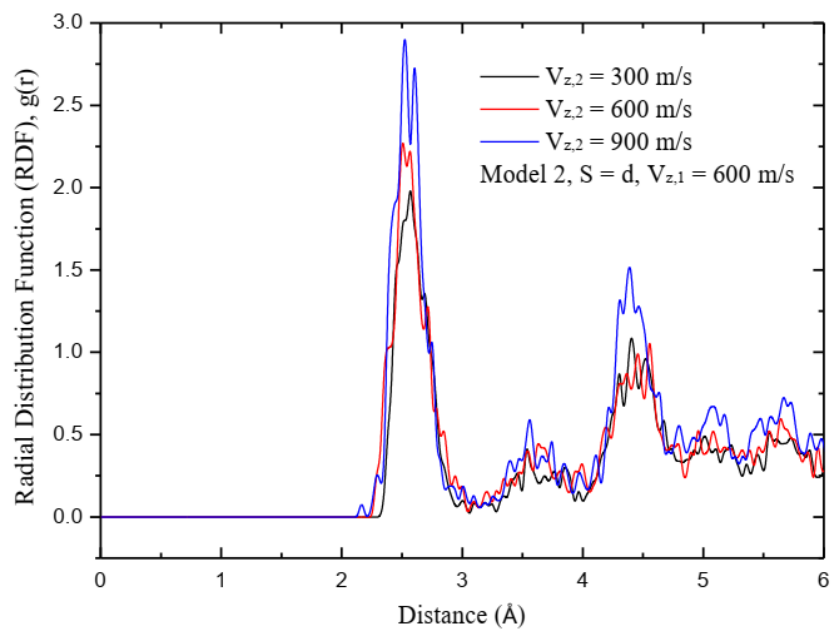

Figure 12. RDFs in Model 2: $V_{1, z}=600 \mathrm{~m} / \mathrm{s}$ and various $V_{2, z}$.

Figure 13a shows that the absolute value of the binding energy between the two clusters tends to be zero, as they have no contact with each other, and the binding energy between any one cluster and the free layer presents a slightly decreasing trend, as the two clusters are far away from each other. The reason is that the weakening thermal-softening effect at the larger $S$ gives rise to the decreasing mechanical interlocking and metallurgic bonding between any one cluster and the substrate. Since the thermal-softening effect from any one cluster to the other one is weakened with the increasing $S$, the substrate deformation also presents a decreasing trend, as can be observed in Figure 13b. Figure 14 shows that the peak values of the RDFs decrease as $S$ becomes larger, thus further verifying the decrease in the metallurgic bonding. 

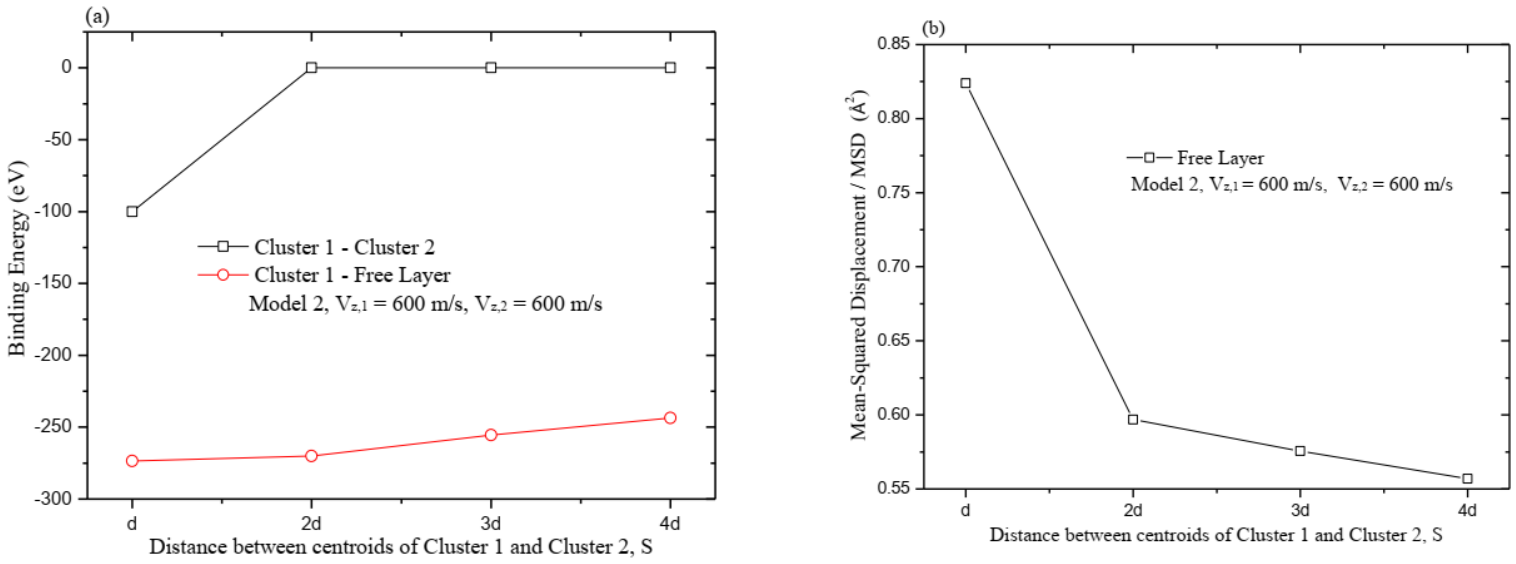

Figure 13. (a) Binding energy between Cluster 1 and Cluster 2 (or free layer) at different $\mathrm{S}$. (b) MSD of free layer at different $\mathrm{S}$. In all cases, $V_{z, 1}=600 \mathrm{~m} / \mathrm{s}$ and $V_{z, 2}=600 \mathrm{~m} / \mathrm{s}$.
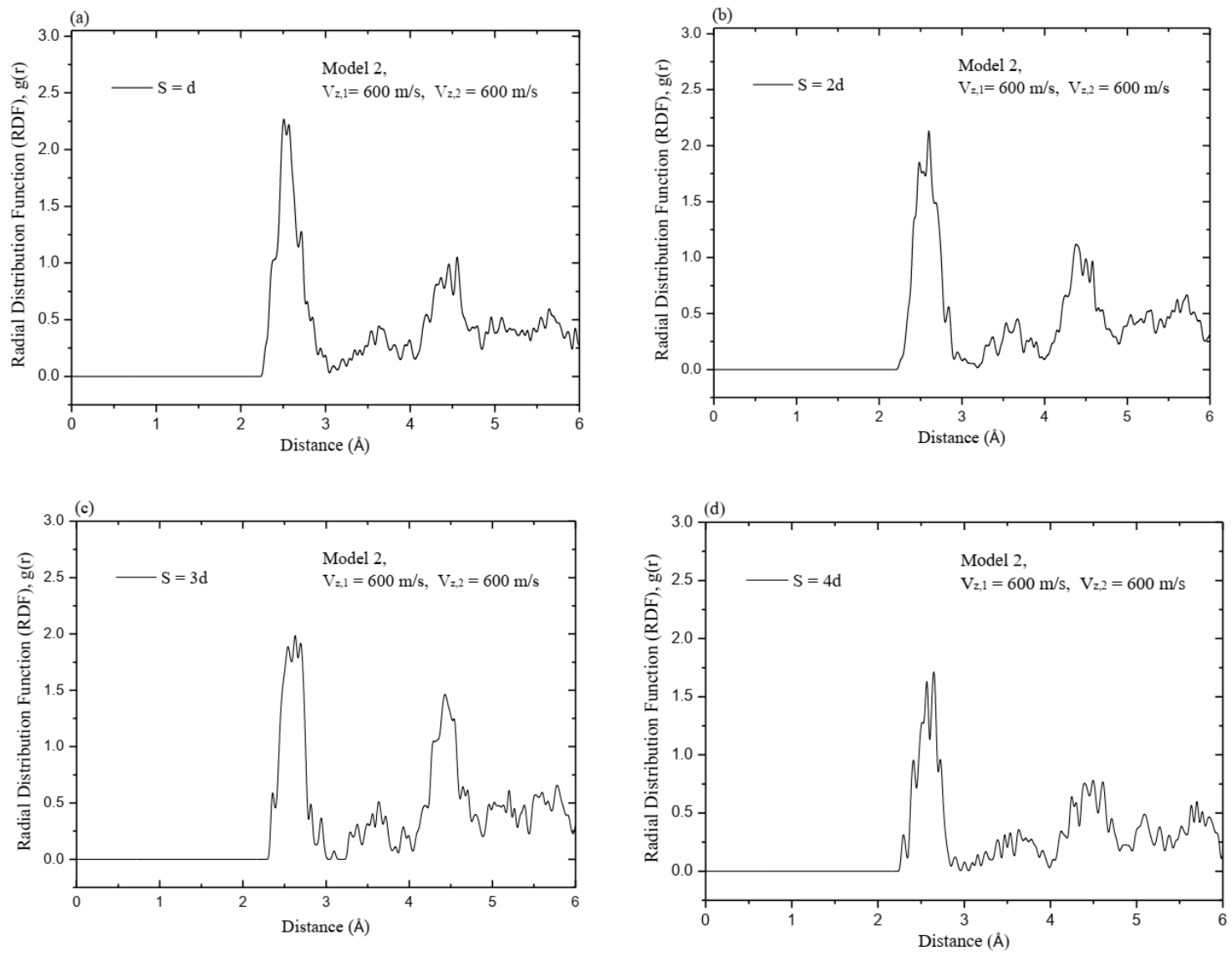

Figure 14. RDFs at $V_{z, 1}=600 \mathrm{~m} / \mathrm{s}$ and $V_{z, 2}=600 \mathrm{~m} / \mathrm{s}$ in Model 2: (a) $\mathrm{S}=\mathrm{d},(\mathbf{b}) \mathrm{S}=2 \mathrm{~d}$, (c) $\mathrm{S}=3 \mathrm{~d}$ and $(\mathbf{d}) S=4 d$.

\subsection{Two Clusters Arranged Vertically at the Different Incident Velocities in Model 3}

As shown in Figure 15a,b, the bonding strength between Cluster 3 and the substrate and between Cluster 4 and the substrate both dramatically enlarge with increasing $V_{z, 3}$ and $V_{z, 4}$. It is attributed to the peening effect from Cluster 3 to Cluster 4 and the thermalsoftening effect from Cluster 4 to Cluster 3 . The bonding strength between Cluster 3 and Cluster 4 slightly decreases as one of $V_{z, 3}$ and $V_{z, 4}$ increases, as is illustrated by the symbol $\square$ in Figure 15a, b. It indicates that the collision between the two clusters arranged vertically for anywhere and anytime can make both of their distances longer because of 
the momentum conservation, which weakens the bonding strength between them. In addition, Cluster 3 must collide against Cluster 4 during the flying course at $V_{z, 3}>600 \mathrm{~m} / \mathrm{s}$ and $V_{z, 4}<600 \mathrm{~m} / \mathrm{s}$, and they only happen to collide with each other on the substrate at $V_{z, 3}<600 \mathrm{~m} / \mathrm{s}$ and $V_{z, 4}>600 \mathrm{~m} / \mathrm{s}$. Comparing Figure $15 \mathrm{a}$ with Figure $15 \mathrm{~b}$, it can be observed that the bonding strength between the clusters and the substrate is larger as the two clusters collide with each other before impacting onto the substrate, and vice versa. The collision during the flying course rises the incident velocity of the first cluster and decreases that of the latter cluster according to the momentum conservation, which shortens the interval time of the two clusters separately impacting the substrate, and then largely weakens the cooling and solidification of the softened substrate. Comparing the symbols $\times$ with + in Figure 15c, we can see that the substrate deformation is smaller as the two clusters impact the substrate without collision with each other during the flying course. It is also attributed to a larger velocity difference between the two clusters that can lead to a longer cooling and solidifying time after the first cluster has arrived at the substrate. Figure 16 shows that the crystal order degree of the whole system is improved at the larger $V_{z, 3}$ and $V_{z, 4}$, resulting in a stronger metallurgic bonding. Comparing Figure $16 \mathrm{a}$ with Figure $16 \mathrm{~b}$, we can see that the RDF values at $V_{z, 3}=900 \mathrm{~m} / \mathrm{s}$ and $V_{z, 4}=600 \mathrm{~m} / \mathrm{s}$ are higher than the ones at $V_{z, 3}=600 \mathrm{~m} / \mathrm{s}$ and $V_{z, 4}=900 \mathrm{~m} / \mathrm{s}$, and the values at $V_{z, 3}=600 \mathrm{~m} / \mathrm{s}$ and $V_{z, 4}=300 \mathrm{~m} / \mathrm{s}$ are higher than the ones at $V_{z, 3}=300 \mathrm{~m} / \mathrm{s}$ and $V_{z, 4}=600 \mathrm{~m} / \mathrm{s}$. It also suggests that the metallurgic bonding is enhanced if the velocity difference between the two clusters reduces through the momentum exchange before impacting onto the substrate.
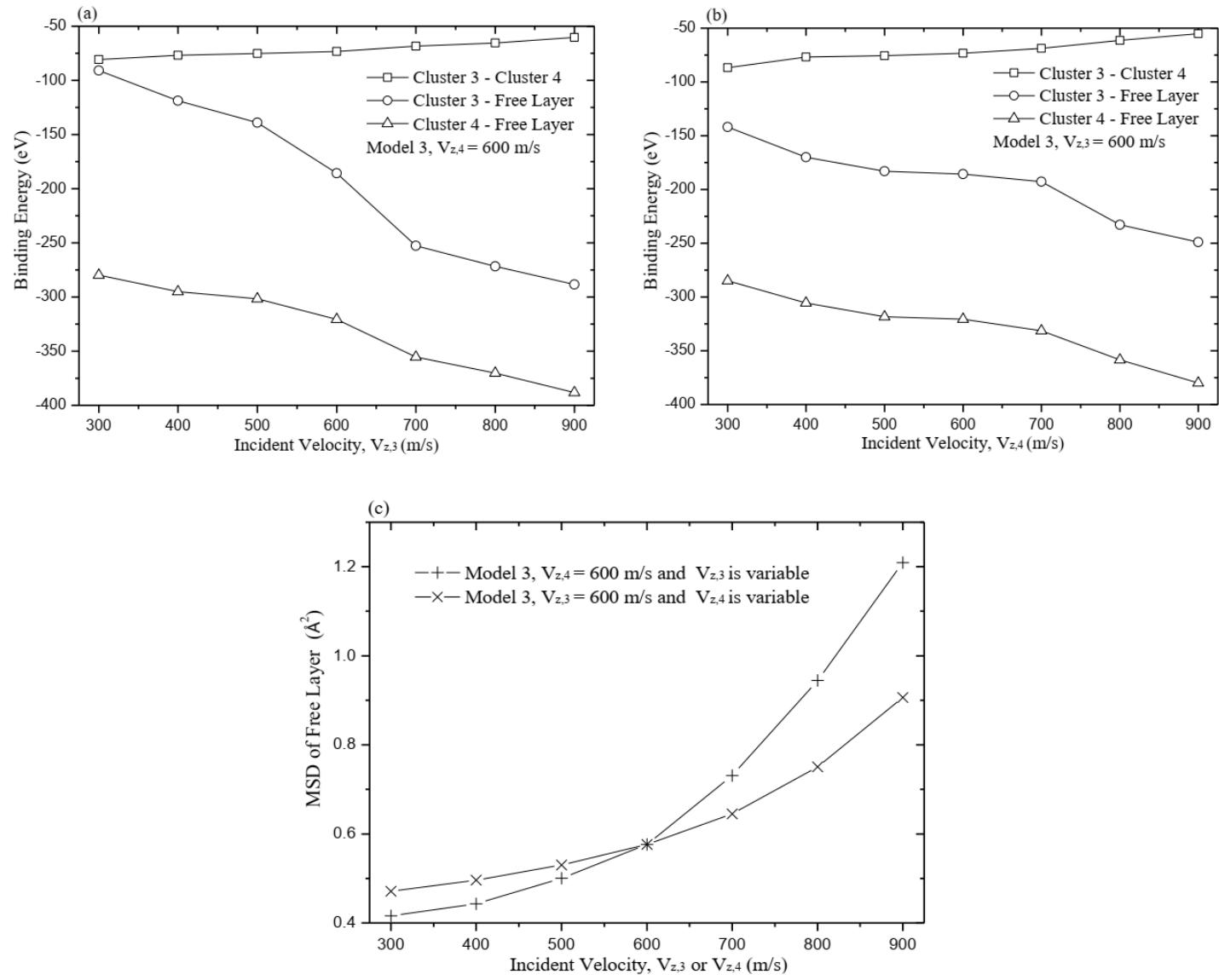

Figure 15. (a) Binding energy among Cluster 3, Cluster 4 and free layer at $V_{z, 4}=600 \mathrm{~m} / \mathrm{s}$ and various $V_{z, 3} ;$ (b) binding energy among Cluster 3 , Cluster 4 and free layer at $V_{z, 3}=600 \mathrm{~m} / \mathrm{s}$ and various $V_{z, 4}$; and (c) MSD of free layer at various $V_{z, 3}$ and $V_{z, 4}$. 

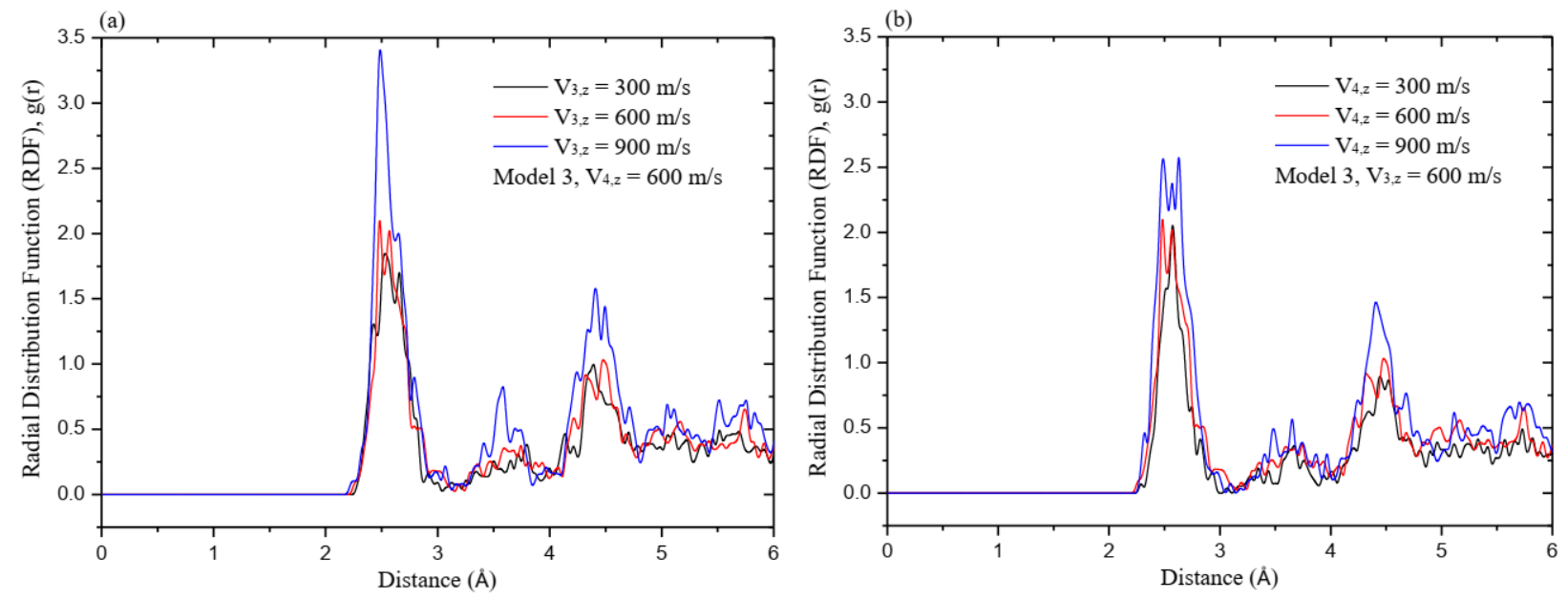

Figure 16. RDFs in Model 3: (a) $V_{z, 4}=600 \mathrm{~m} / \mathrm{s}$ and various $V_{z, 3}$ and (b) $V_{z, 3}=600 \mathrm{~m} / \mathrm{s}$ and various $V_{z, 4}$.

\section{Conclusions}

The deposition characteristics of Pt clusters impacting the Ni substrate in the CGDS process were comprehensively investigated by using MD simulations with three different models. The main conclusions can be summarized as follows:

1. For one single cluster, the normal incident velocity has a great contribution on the depth of the cluster embedding into the substrate, and the tangential incident velocity leads to the scattering on the substrate. The two incident velocities $\left(V_{x, 0}\right.$ and $\left.V_{z, 0}\right)$ and the impact torque $\left(T_{x, 0}\right)$ can all enhance the bonding strength, while $T_{z, 0}$ first decreases and then increases the bonding strength. The enhancements of $V_{z, 0}$ and $T_{x, 0}$ improve the mechanical interlocking and metallurgic bonding, and the enhancements of $V_{x, 0}$ and $T_{z, 0}$ mainly enlarge the mechanical interlocking.

2. For two clusters arranged horizontally, the bonding force between each cluster and the substrate becomes stronger, raising the incident velocity of any one of the two neighboring clusters. The thermal-softening effect of the first cluster impacting on the substrate contributes more to its own metallurgic bonding and the mechanical interlocking of the latter one. The bonding strength is dominantly determined by the mechanical interlocking at $V_{z, 2}<600 \mathrm{~m} / \mathrm{s}$ and the metallurgic bonding at $V_{z, 2}>$ $700 \mathrm{~m} / \mathrm{s}$. When the distance between the two clusters becomes larger, the mechanical interlocking and the metallurgic bonding between any one cluster and the substrate significantly weaken.

3. For two clusters arranged vertically, the peening effect from the upper cluster and the thermal-softening effect from the lower cluster can always enhance the bonding strength between any one cluster and the substrate. If the two vertical clusters happen to collide with each other during the flying course, increasing the incident velocity of the first cluster and decreasing the incident velocity of the latter cluster largely shortens the cooling and solidifying times and enhances the metal interlocking and the metallurgic bonding.

Author Contributions: Conceptualization, X.Z. and X.L.; methodology, X.Z.; X.L. and T.S.; software, T.S.; validation, X.Z. and T.S.; formal analysis, X.L. and T.S.; investigation, X.Z.; X.L. and T.S. resources, X.L. and T.S.; data curation, X.Z.; X.L. and T.S.; writing-original draft preparation, X.Z.; X.L. and T.S.; writing-review and editing, X.Z.; supervision, X.Z.; project administration, X.L. and T.S.; funding acquisition, X.Z. All authors have read and agreed to the published version of the manuscript. 
Funding: The authors are grateful for the financial support provided by the Applied Basic Research Programs of Shanxi Province in China (Grant Number 201801D221370), Scientific and Technological Innovation Programs of Higher Education Institutions (Grant Number 2019L0601) and National Natural Science Foundation of China (Grant Numbers 51,776,027 and 52106063).

Institutional Review Board Statement: Not applicable.

Informed Consent Statement: Not applicable.

Data Availability Statement: This study does not report any other data except for that included in this paper.

Acknowledgments: The authors would like to thank North University and Shanxi Qingze Sunshine Environmental Protection Technology Co. LTD for their supports in this study.

Conflicts of Interest: The authors declare no conflict of interest.

\section{References}

1. Yao, J.; He, Y.; Wang, D.; Peng, H.; Guo, H.; Gong, S. Thermal barrier coatings with (Al2O3-Y2O3)/(Pt or Pt-Au) composite bond coat and 8YSZ top coat on Ni-based superalloy. Appl. Surf. Sci. 2013, 286, 298-305. [CrossRef]

2. Alam, Z.; Sarkar, S.B.; Das, D.K. Refurbishment of thermally degraded diffusion Pt-aluminide (PtAl) bond coat on a Ni-base superalloy. Surf. Coat. Technol. 2018, 354, 101-111. [CrossRef]

3. Assadi, H.; Gärtner, F.; Stoltenhoff, T.; Kreye, H. Bonding mechanism in cold gas spraying. Acta Mater. 2003, 51, 4379-4394. [CrossRef]

4. Li, W.; Liao, H.; Li, C.-J.; Li, G.; Coddet, C.; Wang, X. On high velocity impact of micro-sized metallic particles in cold spraying. Appl. Surf. Sci. 2006, 253, 2852-2862. [CrossRef]

5. Rahmati, S.; Ghaei, A. The Use of Particle/Substrate Material Models in Simulation of Cold-Gas Dynamic-Spray Process. J. Therm. Spray Technol. 2014, 23, 530-540. [CrossRef]

6. Meng, F.; Hu, D.; Gao, Y.; Yue, S.; Song, J. Cold-spray bonding mechanisms and deposition efficiency prediction for particle/substrate with distinct deformability. Mater. Des. 2016, 109, 503-510. [CrossRef]

7. Hussain, T.; McCartney, G.; Shipway, P.H.; Zhang, D. Bonding Mechanisms in Cold Spraying: The Contributions of Metallurgical and Mechanical Components. J. Therm. Spray Technol. 2009, 18, 364-379. [CrossRef]

8. Khodabakhshi, F.; Marzbanrad, B.; Jahed, H.; Gerlich, A. Interfacial bonding mechanisms between aluminum and titanium during cold gas spraying followed by friction-stir modification. Appl. Surf. Sci. 2018, 462, 739-752. [CrossRef]

9. Xie, Y.; Chen, C.; Planche, M.-P.; Deng, S.; Huang, R.; Ren, Z.; Liao, H. Strengthened Peening Effect on Metallurgical Bonding Formation in Cold Spray Additive Manufacturing. J. Therm. Spray Technol. 2019, 28, 769-779. [CrossRef]

10. Xiong, Y.; Xiong, X.; Yoon, S.; Bae, G.; Lee, C. Dependence of Bonding Mechanisms of Cold Sprayed Coatings on Strain-RateInduced Non-Equilibrium Phase Transformation. J. Therm. Spray Technol. 2011, 20, 860-865. [CrossRef]

11. Hussain, T.; Mc Cartney, G.; Shipway, P. Bonding between aluminium and copper in cold spraying: Story of asymmetry. Mater. Sci. Technol. 2012, 28, 1371-1378. [CrossRef]

12. Yin, S.; Suo, X.; Xie, Y.; Li, W.; Lupoi, R.; Liao, H. Effect of substrate temperature on interfacial bonding for cold spray of Ni onto Cu. J. Mater. Sci. 2015, 50, 7448-7457. [CrossRef]

13. Xie, Y.; Planche, M.-P.; Raoelison, R.; Hervé, P.; Suo, X.; He, P.; Liao, H. Investigation on the influence of particle preheating temperature on bonding of cold-sprayed nickel coatings. Surf. Coat. Technol. 2017, 318, 99-105. [CrossRef]

14. Gao, H.; Liu, C.; Song, F.H. Molecular Dynamics Simulation of the Influence Factors of Particle Depositing on Surface during Cold Spray. Adv. Mater. Res. 2013, 652-654, 1916-1924. [CrossRef]

15. Gao, H.; Zhao, L.; Zeng, D.; Gao, L. Molecular Dynamics Simulation of Au Cluster Depositing on Au Surface in Cold Gas Spray. First Int. Conf. Integr. Commer. Micro Nanosyst. Parts A B 2007, 195-202. [CrossRef]

16. Malama, T.; Hamweendo, A.; Botef, I. Molecular Dynamics Simulation of Ti and Ni Particles on Ti Substrate in the Cold Gas Dynamic Spray (CGDS) Process. Mater. Sci. Forum 2015, 828-829, 453-460. [CrossRef]

17. Wang, K.; Liu, J.; Chen, Q. Palladium clusters deposited on the heterogeneous substrates. Appl. Surf. Sci. 2016, 376, 105-112. [CrossRef]

18. Joshi, A.; James, S. Molecular dynamics simulation study of cold spray process. J. Manuf. Process. 2018, 33, 136-143. [CrossRef]

19. Joshi, A.; James, S. Molecular Dynamics Simulation Study on Effect of Process Parameters on Coatings during Cold Spray Process. Procedia Manuf. 2018, 26, 190-197. [CrossRef]

20. Suresh, S.; Lee, S.-W.; Aindow, M.; Brody, H.D.; Champagne, V.K., Jr.; Dongare, A.M. Unraveling the Mesoscale Evolution of Microstructure during Supersonic Impact of Aluminum Powder Particles. Sci. Rep. 2018, 8, 10075. [CrossRef]

21. Jami, H.; Jabbarzadeh, A. Unravelling ultrafast deformation mechanisms in surface deposition of titanium nanoparticles. Appl. Surf. Sci. 2019, 489, 446-461. [CrossRef]

22. Jami, H.; Jabbarzadeh, A. Effect of particle shape on mechanics of impact in the deposition of titanium nanoparticles on a titanium substrate. Surf. Coat. Technol. 2020, 394, 125880. [CrossRef] 
23. Rahmati, S.; Zúñiga, A.; Jodoin, B.; Veiga, R. Deformation of copper particles upon impact: A molecular dynamics study of cold spray. Comput. Mater. Sci. 2020, 171, 109219. [CrossRef]

24. Oyinbo, S.T.; Jen, T.-C.; Zhu, Y.; Ajiboye, J.S.; Ismail, S.O. Atomistic simulations of interfacial deformation and bonding mechanism of Pd-Cu composite metal membrane using cold gas dynamic spray process. Vacuum 2020, 182, 109779. [CrossRef]

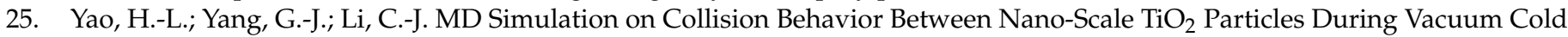
Spraying. J. Nanosci. Nanotechnol. 2018, 18, 2657-2664. [CrossRef]

26. Yao, H.-L.; Hu, X.-Z.; Yang, G.-J. Effect of Particle Size and Impact Velocity on Collision Behaviors Between Nano-Scale TiN Particles: MD Simulation. J. Nanosci. Nanotechnol. 2018, 18, 4121-4126. [CrossRef]

27. Yao, H.-L.; Yang, G.-J.; Li, C.-J. Molecular dynamics simulation and experimental verification for bonding formation of solid-state $\mathrm{TiO}_{2}$ nano-particles induced by high velocity collision. Ceram. Int. 2019, 45, 4700-4706. [CrossRef]

28. Jami, H.; Jabbarzadeh, A. Ultrafast thermomechanical effects in aerosol deposition of hydroxyapatite nanoparticles on a titanium substrate. Surf. Coat. Technol. 2020, 382, 125173. [CrossRef]

29. Jami, H.; Jabbarzadeh, A. Molecular simulation of high-velocity deposition of titanium dioxide nanoparticles on titanium. Appl. Surf. Sci. 2020, 542, 148567. [CrossRef]

30. Li, G.; Wang, X.-F.; Li, W.-Y. Effect of different incidence angles on bonding performance in cold spraying. Trans. Nonferrous Met. Soc. China 2007, 17, 116-121. [CrossRef]

31. Wang, X.; Feng, F.; Klecka, M.A.; Mordasky, M.D.; Garofano, J.K.; El-Wardany, T.; Nardi, A.; Champagne, V.K. Characterization and modeling of the bonding process in cold spray additive manufacturing. Addit. Manuf. 2015, 8, 149-162. [CrossRef]

32. Chen, C.; Xie, Y.; Yin, S.; Planche, M.-P.; Deng, S.; Lupoi, R.; Liao, H. Evaluation of the interfacial bonding between particles and substrate in angular cold spray. Mater. Lett. 2016, 173, 76-79. [CrossRef]

33. Zhu, L.; Jen, T.-C.; Pan, Y.-T.; Chen, H.-S. Particle Bonding Mechanism in Cold Gas Dynamic Spray: A Three-Dimensional Approach. J. Therm. Spray Technol. 2017, 26, 1859-1873. [CrossRef]

34. Fu, S.-L.; Li, C.-X.; Wei, Y.-K.; Luo, X.-T.; Yang, G.-J.; Li, C.-J.; Li, J.-L. Novel Method of Aluminum to Copper Bonding by Cold Spray. J. Therm. Spray Technol. 2018, 27, 624-640. [CrossRef]

35. Braga, C.; Travis, K. A configurational temperature Nosé-Hoover thermostat. J. Chem. Phys. 2005, 123, 134101. [CrossRef]

36. Plimpton, S. Fast Parallel Algorithms for Short-Range Molecular Dynamics. J. Comput. Phys. 1995, 117, 1-19. [CrossRef]

37. Zhou, X.W.; Johnson, R.A.; Wadley, H.N.G. Misfit-energy-increasing dislocations in vapor-deposited CoFe/NiFe multilayers. Phys. Rev. B 2004, 69, 144113. [CrossRef]

38. Chromik, R.R.; Goldbaum, D.; Shockley, J.M.; Yue, S.; Irissou, E.; Legoux, J.-G.; Randall, N.X. Modified ball bond shear test for determination of adhesion strength of cold spray splats. Surf. Coatings Technol. 2010, 205, 1409-1414. [CrossRef]

39. Yildirim, B.; Fukanuma, H.; Ando, T.; Gouldstone, A.; Müftü, S. A Numerical Investigation into Cold Spray Bonding Processes. J. Tribol. 2014, 137, 011102. [CrossRef]

40. Kumar, S.; Bae, G.; Lee, C. Influence of substrate roughness on bonding mechanism in cold spray. Surf. Coat. Technol. 2016, 304, 592-605. [CrossRef] 